\title{
Activation of transient receptor potential vanilloid 4 protects articular cartilage against IL-1 $\beta$-induced inflammatory responses by regulating the CaMKK/AMPK/NF-KB signaling pathway
}

Kyosuke Hattori

Nagoya University Graduate School of Medicine

Nobunori Takahashi ( $\nabla$ nobunori@med.nagoya-u.ac.jp)

Nagoya University Graduate School of Medicine

Kenya Terabe

Nagoya University Graduate School of Medicine

Yoshifumi Ohashi

Nagoya University Graduate School of Medicine

Kenji Kishimoto

Nagoya University Graduate School of Medicine

Yutaka Yokota

Nagoya University Graduate School of Medicine

Mochihito Suzuki

Nagoya University Graduate School of Medicine

Toshihisa Kojima

Nagoya University Graduate School of Medicine

Shiro Imagama

Nagoya University Graduate School of Medicine

\section{Research Article}

Keywords: inflammatory responses, osteoarthritis, transient receptor potential vanilloid 4 (TRPV4)

Posted Date: April 28th, 2021

DOI: https://doi.org/10.21203/rs.3.rs-453677/v1

License: (c) (1) This work is licensed under a Creative Commons Attribution 4.0 International License.

Read Full License 
Version of Record: A version of this preprint was published at Scientific Reports on July 30th, 2021. See the published version at https://doi.org/10.1038/s41598-021-94938-3. 


\section{Abstract}

Transient receptor potential vanilloid 4 (TRPV4) plays an important role in chondrocytes via $\mathrm{Ca}^{2+}$ signaling. However, its role in the progression of osteoarthritis is unclear. This study aimed to evaluate the effects of TRPV4 activation on articular cartilage and chondrocytes stimulated with interleukin (IL)-1ß. Bovine and human articular chondrocytes were stimulated with various agents, including IL-1 $\beta$, GSK1016790A (GSK101; a TRPV4 agonist), Compound C (an AMP-activated protein kinase (AMPK) inhibitor), and STO-609 (a calmodulin-dependent protein kinase kinase (CaMKK) inhibitor), and were processed for Western blot analysis and real-time PCR. The dimethylmethylene blue (DMMB) assay and Safranin 0 staining were also performed. GSK101 reversed the IL-1 $\beta$-induced increase in expression of matrix metalloproteinase (MMP)-13 and decrease in expression of aggrecan. GSK101 also decreased proteoglycan release in the DMMB assay and retained Safranin 0 staining of articular cartilage tissue. Furthermore, GSK101 increased AMPK phosphorylation and decreased IL-1 $\beta$-induced nuclear factor kappa B (NF-KB) phosphorylation. Compound C and STO-609 reversed the suppressive effects of GSK101 on NF-KB activation and MMP-13 expression. In conclusion, TRPV4 activation had chondroprotective effects on articular cartilage stimulated with IL-1 $\beta$ by activating CaMKK/AMPK and suppressing the NF-KB pathway. TRPV activators may offer a promising therapeutic option for preventing the progression of osteoarthritis.

\section{Introduction}

Transient receptor potential vanilloid 4 (TRPV4), an osmotically active ion channel associated with $\mathrm{Ca}^{2+}$ intake, plays an important role in mechano-transduction pathways of chondrocytes via $\mathrm{Ca}^{2+}$ signaling $^{1-3}$. However, the role of TRPV4 in the progression of osteoarthritis (OA) is controversial. For instance, previous studies have shown that TRPV4 activation induced both catabolic and anabolic responses in chondrocytes in vitro ${ }^{3-5}$. Similarly, inconsistent results have been reported in TRPV4-knockout mice in vivo, with one study reporting progression of $O A$ and another reporting a reduction of $O A$ in these mice ${ }^{6,7}$.

We previously reported that TRPV4 stimulated with GSK101 plays a role in chondrogenesis by inducing the expression of chondrogenic markers including sex-determining region Y-box transcription factor (SOX9) and aggrecan (AGC) ${ }^{8}$, whereas signaling pathways of up-regulation of SOX9 and AGC via activation of TRPV4 was not well shown. It has been reported that $\mathrm{Ca}^{2+}$ intake activates AMP-activated protein kinase (AMPK), an evolutionarily conserved fuel and stress-sensing enzyme that can be activated by calmodulin-dependent protein kinase kinase-2 (CAMKK2) and that AMPK activation suppresses matrix degradation responses to IL-1 $\beta$ in chondrocytes ${ }^{9,10}$. Consistent with this, some drugs have been reported to attenuate cartilage degeneration by activating $A \mathrm{MPK}^{11-14}$. Although regulation of the CaMKK/AMPK/NF-KB signaling pathway inhibits inflammation, which plays a role in modern chronic diseases such as diabetes and cancer ${ }^{15}$, the role of this pathway in articular cartilage degradation and OA progression is unknown. 
Based on the mechanistic findings discussed above, we hypothesized that TRPV4 may have a chondroprotective effect against arthritis caused by IL-1 $\beta$ stimulation. To test this, the present study aimed to determine whether TRPV4 activation in chondrocytes protects articular cartilage from degradation and inhibits the progression of OA via the CaMKK/AMPK/NF-KB signaling pathway.

\section{Results}

\section{Determination of appropriate GSK101 concentration to inhibit IL-1 $\beta$-induced cartilage degradation}

GSK101 was used as a specific TRPV4 agonist to explore the effects of TRPV4 activation on the procatabolic phenotype of activated chondrocytes. In an MTS assay, GSK101 was not cytotoxic to BACs and HACs at concentrations of up to 1000 pM, but was cytotoxic at 10000 pM (Figure 1a). In BACs, GSK101 significantly reversed the IL-1 $\beta$-induced increase in expression of MMP13 mRNA and decrease in expression of AGC and SOX9 mRNA in a dose-dependent manner (Figure 1b). Since GSK was most effective at a concentration of $1000 \mathrm{pM}$, this concentration was used for subsequent experiments.

\section{TRPV4 activation inhibits IL-1 $\beta$-induced cartilage degradation}

When examined by Western blot and densitometry, GSK101 at 1000 pM significantly reduced the expression of MMP13 induced by IL-1 $\beta$ stimulation in HACs (Figure 2a). To determine whether the addition of GSK101 can block proteoglycan release from bovine articular cartilage explants treated with IL-1 $\beta$ ex vivo, a DMMB colorimetric assay of SGAG release was performed. Stimulating full-thickness 4$\mathrm{mm}$ cores of cartilage explants with IL-1 $\beta$ significantly increased the elution of sGAG into the medium compared to the untreated control. Co-treatment with GSK101 significantly suppressed the IL-1 $\beta$-induced release of SGAG (Figure 2b). In Safranin O/Fast Green staining of bovine articular cartilage explant cores after 7 days of IL-1 $\beta$ stimulation, a substantial amount of proteoglycans was lost from the explant. This effect was almost completely rescued by co-treatment with GSK101 (Figure 2c).

\section{Pathways involved in chondroprotective effect of TRPV4 activation}

To identify signaling pathways which may be involved in the chondroprotective effect of GSK101, BACs were stimulated with IL-1 $\beta$ in the absence or presence of GSK101. Western blot and band densitometry analyses revealed that stimulation with IL-1 $\beta$ for 30 min increased the levels of phospho-(p)NF-KB, and GSK101 treatment for 30 min enhanced the levels of pAMPK, compared with untreated control samples (Figures $3 \mathrm{a}$ and $3 \mathrm{~b}$ ). Co-treatment with GSK101 significantly suppressed IL-1 1 -induced NF-KB phosphorylation.

Compound $\mathrm{C}$, a chemical inhibitor of AMPK phosphorylation, was used to determine whether the phosphorylation of AMPK is involved in the GSK101-mediated suppression of IL-1 $\beta$-induced NF-KB phosphorylation (Figure 3a). Pre-treatment with compound $C$ significantly suppressed the phosphorylation of AMPK $(p=0.016)$ and increased the phosphorylation of NF-KB $(p=0.017)$ compared to cells treated with IL-1 $\beta$ and GSK101. 
ST0-609, an inhibitor of CaMKK activation, was used to determine whether CaMKK activation crosstalked with the AMPK/NF-KB pathway (Figure 3b). Pre-treatment with STO-609 for 1 hour significantly suppressed the phosphorylation of AMPK observed with the combination of GSK101 and IL-1 $\beta(p<0.001)$, and also countered the suppressive effect of GSK101 on IL-1 $\beta$-induced NF-KB phosphorylation ( $p=0.021)$.

These results collectively suggest that TRPV4 activation suppresses IL-1 $\beta$-induced NF-KB activation by activating the CaKMM/AMPK pathway.

\section{TRPV4-mediated suppression of IL-1 $\beta$-induced MMP-13 expression via the CaMKK/AMPK/NF-KB pathway}

As discussed above, the TRPV4/CaMKK/AMPK pathway is involved in suppressing IL-1 $\beta$-induced NF-KB activation. Thus, we examined whether this mechanism was involved in the chondroprotective effect of TRPV4.

Both MMP-13 mRNA expression in BACs $(n=3)$ and MMP-13 protein expression in HACs (since the antiMMP13 antibody did not react with bovine MMP13) under various conditions were examined. Pretreatment with STO-609 countered the suppressive effect of GSK101 on IL-1 $\beta$-induced expression of MMP13 mRNA ( $p<0.001$; Figure 4a). Similar effects of ST0-609 were observed on MMP13 protein levels, albeit to a lesser extent ( $p=0.044$; Figure $4 b$ ). These results suggest that activation of CaMKK by TRPV4 is involved in the chondroprotective effect of TRPV4 against IL-1 $\beta$-induced cartilage degradation.

\section{Discussion}

Although recent studies have found that drugs such as metformin and protectin DX attenuate cartilage damage via the AMPK/NF-KB pathway ${ }^{12-14}$, the underlying mechanism was unclear. In addition, while the CaMKK/AMPK pathway has been reported to play an important role in myocytes and in circumventricular $\operatorname{organs}^{16,17}$, the role of this pathway in chondrocytes was unknown. The present study is the first to demonstrate that TRPV4 activation inhibits NF-KB phosphorylation by activating the CaMKK/AMPK pathway in chondrocytes in the presence of IL-1 $\beta$, resulting in a chondroprotective effect.

Jeon et al. previously reported that exercise and contraction induce AMPK activation and inhibit NF-KB activation by increasing the AMP/ATP ratio and/or through the $\mathrm{Ca}^{2+} / \mathrm{CaMKK}$ signaling pathway in the context of diabetes and cancer $^{15}$. Moreover, while moderate cyclic tensile strain suppresses IL-1 $\beta$-induced inflammatory responses in chondrocytes via the AMPK/NF-KB pathway by increasing the AMP/ATP ratio $^{18}$, no study has reported on the role of the CaMKK/AMPK/NF-KB pathway in chondrocytes. We found that TRPV4 activation down-regulated the expression of MMP-13 by activating CaMKK and AMPK and inactivating NF-KB. TRPV4 activation also upregulated the expression of cartilage phenotypic genes, including SOX9 and AGC. Given the role of IL-1 $\beta$ in OA progression by inducing catabolic responses and inhibiting anabolic responses ${ }^{19-22}$, our findings suggest that TRPV4 activation by mechanical stress or chemicals may protect articular cartilage from degeneration and OA progression induced by IL-1 $\beta$. 
The role of TRPV4 in OA has been controversial. In vitro, some studies reported that TRPV4 activation induces catabolic responses in chondrocytes (e.g., increasing the expression of ADAM10 and apoptosis of chondrocytes) ${ }^{4,5}$, while others reported the anabolic effects of TRPV4 activation (e.g., increasing the expression of type 2 collagen and decreasing the expression of disintegrin and metalloproteinase with thrombospondin motifs-5) ${ }^{3}$. This inconsistency has been noted in vivo as well. For instance, while one study reported that male TRPV4 knockout mice exhibited early and severe development of age-related $\mathrm{OA}^{11}$, another reported that the knockout mice were protected from age-related $\mathrm{OA}$, but not from $\mathrm{OA}$ caused by destabilization of the medial meniscus ${ }^{7}$. In the present study, we observed the anabolic effects of TRPV4 activation, as reflected in the increased expression of SOX9 and AGC and decreased expression of MMP-13, as well as the preservation of proteoglycans in articular cartilage tissue in the IL-1 $\beta$ stimulation model. Given the possibility that the effects of TRPV4 activation may differ by experimental model, further confirmatory studies will be needed.

The inconsistent effects of TRPV4 activation in chondrocytes discussed above may reflect differences in the mechanism of AMPK activation. Indeed, AMPK is known to be activated by various mechanisms, including canonical and non-canonical pathways. As we have shown here, in one non-canonical pathway, AMPK is activated via a $\mathrm{Ca}^{2+} / \mathrm{CaMKK} 2$-dependent mechanism. Another non-canonical pathway in which AMPK is activated by glucose starvation was recently reported by Li et al. ${ }^{23}$. In that study, endoplasmic reticulum-localized TRPVs channels and $\mathrm{Ca}^{2+}$ release were inhibited by fructose-1,6-bisphosphate (FBP)unoccupied aldolase under low glucose conditions, subsequently leading to the formation, phosphorylation, and activation of an AXIN-LKB1-AMPK complex on the lysosomal membrane. Importantly, GSK101 inhibited AMPK activation under glucose starvation conditions due to an increase in local $\mathrm{Ca}^{2+}$ concentrations, suggesting that the concentration of the TRPV agonist may lead to differing results because high concentrations can induce a bulk, global increase in $\mathrm{Ca}^{2+}$ concentration via a CaMKK2-dependent mechanism ${ }^{23}$. These results suggest that the effect of TRPV4 activation may depend on various factors, for example, the type of cell, the degree of inflammation, and glucose conditions.

The mechanism underlying the inhibition of NF-KB phosphorylation by AMPK activation remains unclear. Previous studies have reported that activation of AMPK or sirtuin (SIRT) 1 inhibited IL- $1 \beta$-induced inflammatory responses by inhibiting NF-KB activation in chondrocytes ${ }^{11,24,25}$. The activation of peroxisome proliferator activated receptor $y$ coactivator (PGC)-1a and Forkhead box 0 (FOXO)3a by AMPK activation was also reported to inhibit NF-KB activation and inflammatory cytokine-induced catabolic responses in chondrocytes ${ }^{26}$. Given that AMPK activation was reported to induce PGC-1a activation directly or via SIRT1 activation in myocytes ${ }^{16}$, the AMPK/SIRT1/PGC-1a pathway may play a role in suppressing IL-1 $\beta$-induced inflammatory responses by inhibiting NF-KB activation in chondrocytes as well. The link between AMPK activation and suppression of NF-KB may also involve changes in glucose metabolism. In this regard, we recently reported that aerobic respiration switched to glycolysis in 
IL-1 $\beta$-stimulated chondrocytes, and that IL-1 $\beta$ reduced the phosphorylation of AMPK, which was rescued by a chemical glycolysis inhibitor ${ }^{10}$.

In conclusion, the activation of TRPV4 suppressed IL-1 $\beta$-induced chondro-degenerative changes and MMP-13 expression in chondrocytes by activating CaMKK/AMPK and suppressing the activation of NFKB. TRPV activators may offer a promising therapeutic option for preventing OA progression.

\section{Methods}

\section{Cells and cell culture}

Bovine articular chondrocytes (BACs) were isolated from full-thickness slices of the articular surface of metatarsophalangeal joints of young adult cows (aged 18-24 months) which were obtained from Nagoya City Central Wholesale Market in Japan with institutional approval. No live animals were used in this study. HACs were isolated from slices of knee joints of patients who underwent total knee arthroplasty with institutional IRB approval (Ethics Committee of the Nagoya University Graduate School of Medicine \#2020-0146). A written informed consent was obtained from the participants. with the World Medical Association of Helsinki Ethical Principles for Medical Research Involving Human Subjects. Also, these tissues were obtained with no identifying information except age/sex. All methods were carried out in accordance with relevant guidelines and regulations. Slices of bovine and human articular cartilage were digested in $0.2 \%$ Pronase ( $\geq 70,000$ proteolytic units/g dry weight, Catalog \#: 537088; Merck, Germany) for 1 hour at $37^{\circ} \mathrm{C}$ and subsequently in $0.025 \%$ collagenase $\mathrm{P}(>1.5 \mathrm{U} / \mathrm{mg}$ lyophilizate; Catalog \#: 11213865001; Roche, Germany) overnight at $37^{\circ} \mathrm{C}^{27}$. Isolated cells were cultured in Dulbecco's Modified Eagle's Medium (DMEM) low glucose medium with $4 \%$ fetal bovine serum (FBS) and $1 \%$ antibiotics at $37^{\circ} \mathrm{C}$ in a $5 \% \mathrm{CO}_{2}$ environment. After $48-72$ hours in culture, cells were passaged once (P1) and $1-2 \times 10^{5}$ cells $/ \mathrm{cm}^{2}$ were cultured on 6- or 12 -well plates. After static incubation for $48-72$ hours in $4 \%$ FBScontaining medium, cells were cultured in serum-free medium for 12 hours. Subsequently, cells were stimulated in the presence of various agents, including IL-1 $\beta(10 \mathrm{ng} / \mathrm{ml})$, various concentrations of GSK101 (a selective TRPV4 agonist), Compound C (an AMPK inhibitor; $25 \mu \mathrm{M}$ for BACs and $10 \mu \mathrm{M}$ for HACs), and STO-609 (a CaMKK inhibitor; $5 \mu \mathrm{M}$ ), under serum-free conditions. Cells were collected after stimulation and processed for Western blot analysis and real-time PCR.

\section{Cartilage explant cultures}

Full-thickness 4-mm cores of bovine articular cartilage were cultured in $1.0 \mathrm{ml}$ DMEM low glucose medium with $4 \%$ FBS for 24 hours. The medium was then replaced, and tissues were incubated with IL-1 $\beta$ $(10 \mathrm{ng} / \mathrm{ml})$, with or without GSK101. On day 3 of culture, aliquots of medium were analyzed by the dimethylmethylene blue (DMMB) colorimetric assay to measure sulfated glycosaminoglycan (sGAG) release. On day 7 , the treated explants were fixed with $4 \%$ buffered paraformaldehyde overnight at $4^{\circ} \mathrm{C}$ for histology; rinsed in 30\% sucrose/PBS; and embedded in paraffin. Sections $(8 \mu \mathrm{m})$ were prepared and stained with Safranin 0 for the detection of proteoglycans and counterstained with Fast Green ${ }^{10}$. 


\section{Real-time PCR}

Total RNA was extracted with the RNeasy Mini Kit (Qiagen, Germany). Reverse transcription (RT) was performed using the High Capacity cDNA Reverse Transcription Kit (Applied Biosystems, USA). Real time RT-PCR was carried out using a Light cycler System with FastStart Master SYBR Green PLUS (Roche, USA ${ }^{27}$. Primers for matrix metalloproteinase (MMP)-13, AGC, SOX9, and 18S ribosomal RNA (18S rRNA) were synthesized by Sigma-Aldrich (USA). The following primers were used: MMP-13, forward primer 5'TCCAGTTTGCAGAGAGCTACCT-3', reverse primer 5'-CCTGTCAATCACAGAGCTTGCT-3'; AGC, forward primer 5'-AAATATCACTGAGGGTGAAGCCCG-3', reverse primer 5'-ACTTCAGGGACAAACGTGAAAGGC-3'; SOX9, forward primer 5'-CGACTCCCCACATTCCTCCTC-3', reverse primer 5'-

GGACCCTGAGATTGCCCAGAG-3'; and 18S rRNA, forward primer 5'-GTAACCCGTTGAACCCCATT-3', reverse primer 5'-CCATCCAATCGGTAGTAGCG-3'.

\section{Western blot analysis}

The expression of MMP-13, AGC, AMPK, and NF-KB was evaluated by Western blot analysis using BAC and HAC lysates. Cells cultured on 6-well plates were trypsinized and pelleted by centrifugation. Total protein was extracted from cell pellets with Cell Lysis Buffer (Cell Signaling, USA) containing a protease and phosphatase inhibitor cocktail. Samples were separated by $10 \%$ SDS-PAGE under reducing conditions and transferred to a nitrocellulose membrane. Antibodies against MMP-13 (18165-1-AP, Proteintech Group), AGC (ab3778, abcam), pAMPK (2535, Cell Signaling), AMPK (5831, Cell Signaling), pNF-kB (3033, Cell Signaling), NF-кB (8242, Cell Signaling), and beta-actin (4970, Cell Signaling) were used. Band intensities were captured with a digital image scanner and quantified using densitometry software (CS Analyzer 3.0; ATTO, Tokyo, Japan).

\section{Statistical Analysis}

Values are expressed as mean \pm standard deviation (SD). One-way ANOVA with Tukey's test was performed for comparisons. Statistical significance was defined as $p<0.05$. All analyses were performed with BellCurve for Excel version 3.21.

\section{Declarations}

\section{Acknowledgments}

The work was supported in part by a grant from JSPS KAKENHI JP20K18061 and 19K09620 (KT and NT). We also thank to Dr. Tsuyoshi Nishiume, Dr. Yasumori Sobue, Dr. Masataka Maeda and Dr. Daisuke Kihira for the technical supports.

\section{Author contributions}

K.H., N.T., K.T. and T.K. contributed to the conception and design of the study, or acquisition of data, or analysis and interpretation of data. N.T., Y.O., K.K., Y.Y., M.S., K.T. and T.K. contributed to drafting the 
article or revising it critically for important intellectual content. N.T., K.T., T.K. and S.I. contributed to final approval of the version to be submitted.

\section{Competing interests}

The authors declare no conflicts of interest with the contents of this article.

\section{Data availability}

All data generated or analyzed during this study are includes in this published article.

\section{References}

1. Mobasheri A, et al. The chondrocyte channelome: A narrative review. Joint Bone Spine. 86, 29-35. doi: 10.1016/j.jbspin.2018.01.012 (2019).

2. Lv M, et al. Calcium signaling of in situ chondrocytes in articular cartilage under compressive loading: Roles of calcium sources and cell membrane ion channels. J Orthop Res. 36, 730-738. doi: 10.1002/jor.23768 (2018).

3. O'Conor CJ, Leddy HA, Benefield HC, Liedtke WB, Guilak F. TRPV4-mediated mechanotransduction regulates the metabolic response of chondrocytes to dynamic loading. Proc Natl Acad Sci U S A. 28, 1316-1321. doi: 10.1073/pnas.1319569111 (2014).

4. Kobayakawa $T$, et al. Mechanical stress loading induces CD44 cleavage in human chondrocytic HCS2/8 cells. Biochem Biophys Res Commun. 23, 1230-1235. doi: 10.1016/j.bbrc.2016.08.099 (2016).

5. Xu B, et al. Excessive mechanical stress induces chondrocyte apoptosis through TRPV4 in an anterior cruciate ligament-transected rat osteoarthritis model. Life Sci. 1, 158-166. doi: 10.1016/j.Ifs.2019.05.003 (2019).

6. Clark AL, Votta BJ, Kumar S, Liedtke W, Guilak F. Chondroprotective role of the osmotically sensitive ion channel transient receptor potential vanilloid 4: age- and sex-dependent progression of osteoarthritis in Trpv4-deficient mice. Arthritis Rheum. 62, 2973-2983. doi: 10.1002/art.27624 (2010).

7. O'Conor CJ, et al. Cartilage-Specific Knockout of the Mechanosensory Ion Channel TRPV4 Decreases Age-Related Osteoarthritis. Sci Rep. 8, 6:29053. doi: 10.1038/srep29053 (2016).

8. Ogawa Y, et al. Hyaluronan promotes TRPV4-induced chondrogenesis in ATDC5 cells. PLoS One. 8, e0219492. doi: 10.1371/journal.pone.0219492 (2019).

9. Hawley SA, et al. 5'-AMP activates the AMP-activated protein kinase cascade, and Ca2+/calmodulin activates the calmodulin-dependent protein kinase I cascade, via three independent mechanisms. J Biol Chem. 10, 27186-91. doi: 10.1074/jbc.270.45.27186 (1995). 
10. Terabe K, et al. Chondroprotective effects of 4-methylumbelliferone and hyaluronan synthase-2 overexpression involve changes in chondrocyte energy metabolism. J Biol Chem. 22, 17799-17817. doi: 10.1074/jbc.RA119.009556 (2019).

11. Terkeltaub R, Yang B, Lotz M, Liu-Bryan R. Chondrocyte AMP-activated protein kinase activity suppresses matrix degradation responses to proinflammatory cytokines interleukin-1 $\beta$ and tumor necrosis factor a. Arthritis Rheum. 2011 63, 1928-1937. doi: 10.1002/art.30333 (2011).

12. Li J, et al. Metformin limits osteoarthritis development and progression through activation of AMPK signalling. Ann Rheum Dis. 79, 635-645. doi: 10.1136/annrheumdis-2019-216713 (2020).

13. Feng $X$, et al. Metformin attenuates cartilage degeneration in an experimental osteoarthritis model by regulating AMPK/mTOR. Aging (Albany NY). 16, 1087-1103. doi: 10.18632/aging.102635 (2020).

14. Piao S, et al. Protectin DX attenuates IL-1 $\beta$-induced inflammation via the AMPK/NF-KB pathway in chondrocytes and ameliorates osteoarthritis progression in a rat model. Int Immunopharmacol. 78, 106043. doi: 10.1016/j.intimp.2019.106043 (2020).

15. Jeon SM. Regulation and function of AMPK in physiology and diseases. Exp Mol Med. 15, e245. doi: 10.1038/emm.2016.81 (2016).

16. Iwabu $\mathrm{M}$, et al. Adiponectin and AdipoR1 regulate PGC-1alpha and mitochondria by $\mathrm{Ca}(2+)$ and AMPK/SIRT1. Nature. 29, 1313-1319. doi: 10.1038/nature08991 (2010).

17. Yang F, et al. Adropin Is a Key Mediator of Hypoxia Induced Anti-Dipsogenic Effects via TRPV4CamKK-AMPK Signaling in the Circumventricular Organs of Rats. Front Mol Neurosci. 20, 105. doi: 10.3389/fnmol.2017.00105 (2017).

18. Yang $Y$, et al. Mechanical stress protects against osteoarthritis via regulation of the AMPK/NF-KB signaling pathway. J Cell Physiol. 234, 9156-9167. doi: 10.1002/jcp.27592 (2019).

19. Kapoor M, Martel-Pelletier J, Lajeunesse D, Pelletier JP, Fahmi H. Role of proinflammatory cytokines in the pathophysiology of osteoarthritis. Nat Rev Rheumatol. 7, 33-42. doi: 10.1038/nrrheum.2010.196 (2011).

20. Schulze-Tanzil G. Activation and dedifferentiation of chondrocytes: implications in cartilage injury and repair. Ann Anat. 191, 325-338. doi: 10.1016/j.aanat.2009.05.003 (2009).

21. Speichert S, et al. Role of Norepinephrine in IL-1 $\beta$-Induced Chondrocyte Dedifferentiation under Physioxia. Int J Mol Sci. 11, 1212. doi: 10.3390/ijms20051212 (2019).

22. Kour S, et al. IL-3 Decreases Cartilage Degeneration by Downregulating Matrix Metalloproteinases and Reduces Joint Destruction in Osteoarthritic Mice. J Immunol. 15, 5024-5035. doi: 10.4049/jimmunol.1500907 (2016). 
23. Li M, et al. Transient Receptor Potential V Channels Are Essential for Glucose Sensing by Aldolase and AMPK. Cell Metab. 3, 508-524.e12. doi: 10.1016/j.cmet.2019.05.018 (2019).

24. Matsushita T, et al. The overexpression of SIRT1 inhibited osteoarthritic gene expression changes induced by interleukin-1 $\beta$ in human chondrocytes. J Orthop Res. 31, 531-537. doi: 10.1002/jor.22268 (2013).

25. Lei $\mathrm{M}$, et al. Resveratrol inhibits interleukin $1 \beta$-mediated inducible nitric oxide synthase expression in articular chondrocytes by activating SIRT1 and thereby suppressing nuclear factor-KB activity. Eur J Pharmacol. 15, 73-79. doi: 10.1016/j.ejphar.2011.10.015 (2012).

26. Zhao X, et al. Peroxisome proliferator-activated receptor $y$ coactivator $1 \mathrm{a}$ and FoxO3A mediate chondroprotection by AMP-activated protein kinase. Arthritis Rheumatol. 66, 3073-3082. doi: 10.1002/art.38791 (2014).

27. Sobue Y, et al. Inhibition of CD44 intracellular domain production suppresses bovine articular chondrocyte de-differentiation induced by excessive mechanical stress loading. Sci Rep. 17, 14901. doi: 10.1038/s41598-019-50166-4 (2019).

\section{Figures}


(a)
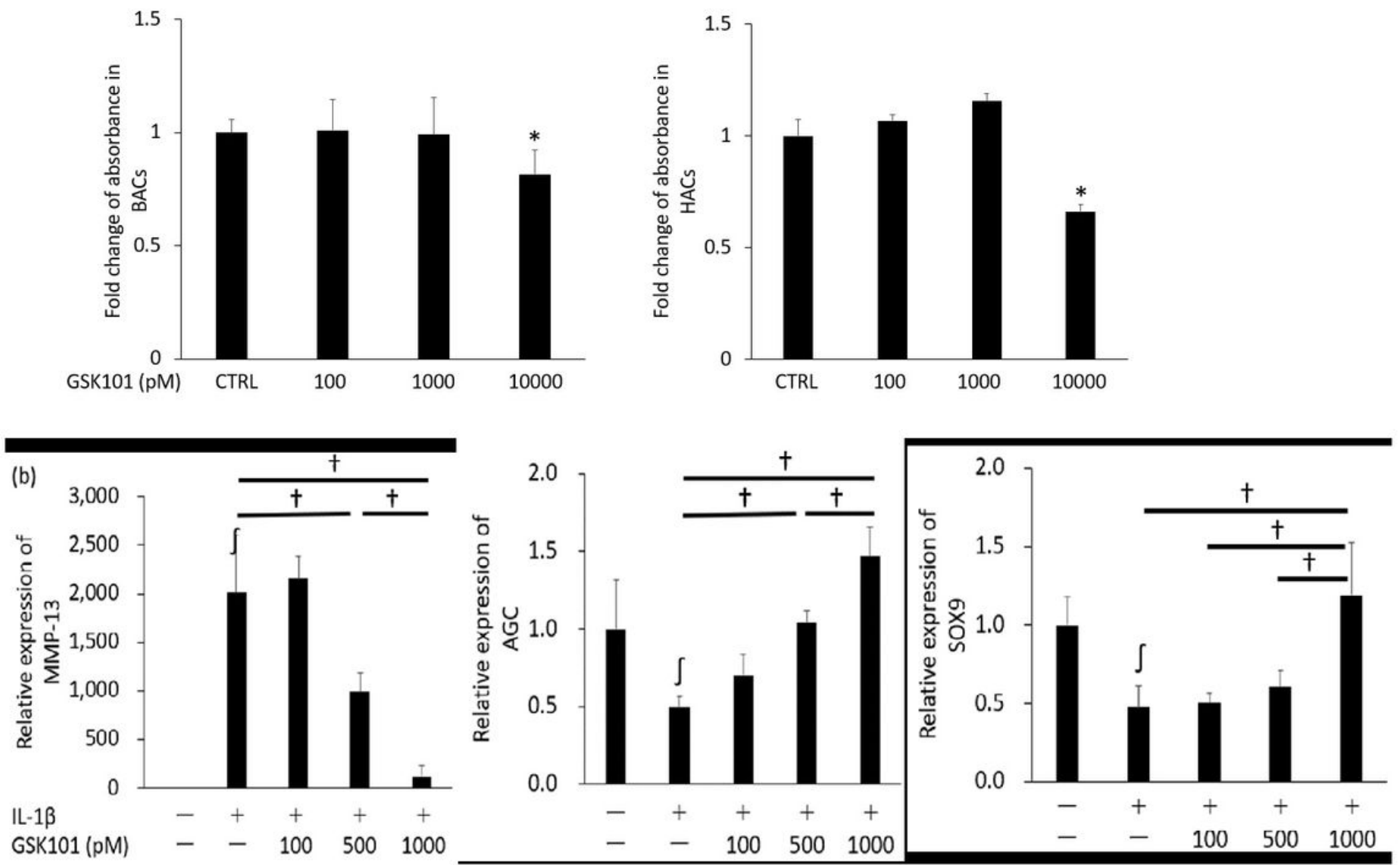

Figure 1

Optimization of GSK101 concentration The optimal concentration of GSK101 for experiments was determined to be 1000 pM based on (a) 48-hour MTS assays in BACs and HACs, and (b) real-time PCRdetermined expression levels of MMP-13, aggrecan, and SOX9 12 hours after treatment in BACs. Experiments were repeated three times $(n=3)$. ${ }^{\star} p<0.05$ compared to untreated control, $\int p<0.05$ compared to untreated control, $+p<0.05$ using one-way ANOVA with Tukey's test. BAC: bovine articular cell; HAC: human articular cell. 
(a)

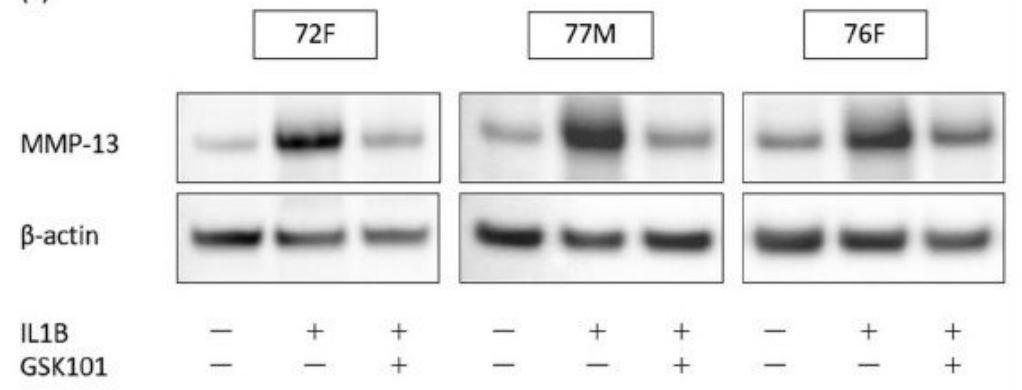

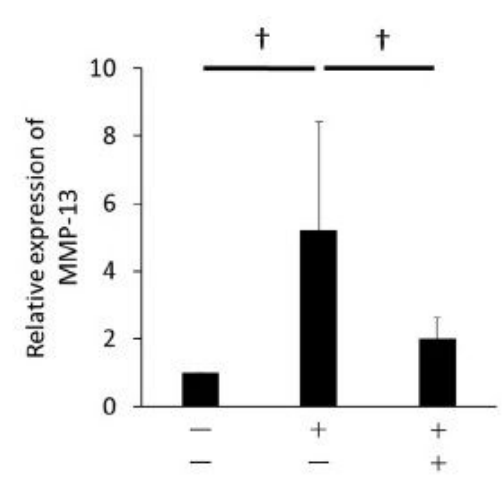

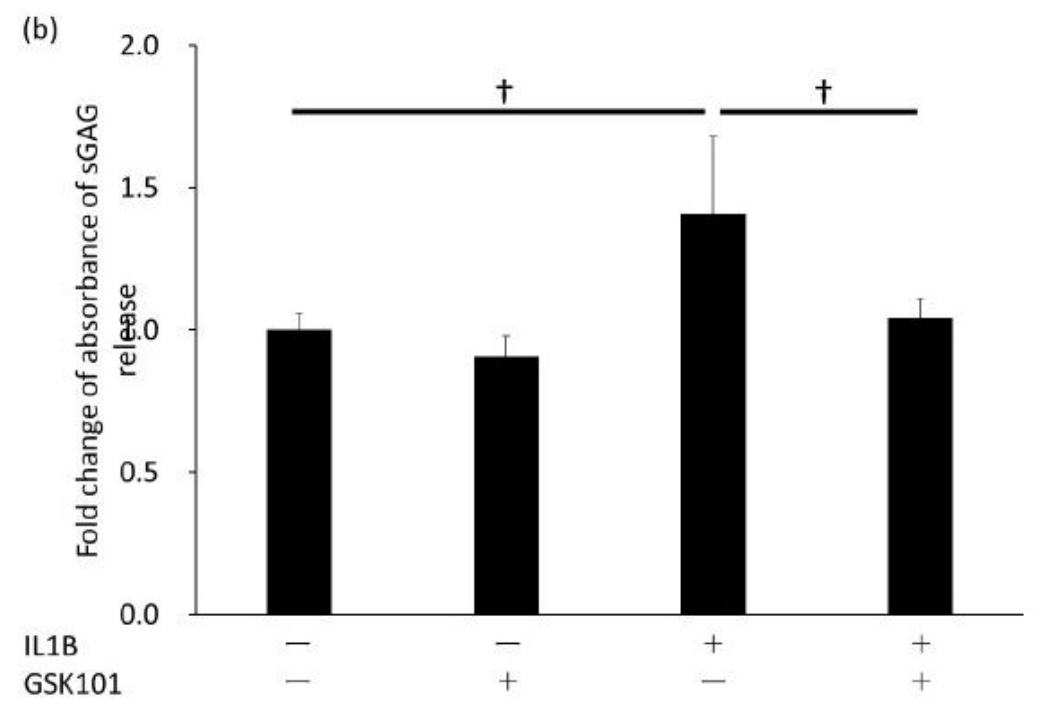

(c)
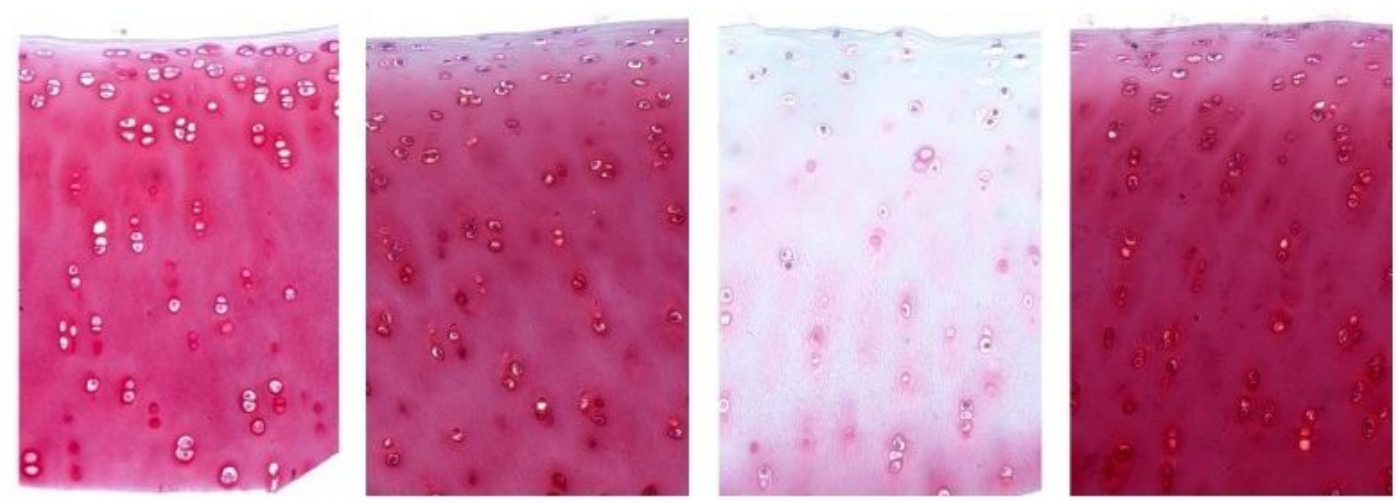

IL-1 $\beta$

GSK101

$-$

$-$

$+$

$+$

$+$

\section{Figure 2}

Reduction of IL-1ß-induced cartilage damage by GSK101 (a) Western blot analysis of HAC lysates to examine the relative expression of MMP-13 after 48 hours of treatment with 1000 pM GSK101.

Experiments were repeated three times $(n=3)$. Each group of MMP-13 and $\beta$-actin was derived from the same membrane. (b) DMMB assay of sGAG release on day 3 of explant culture and (c) Safranin O/Fast Green staining on day 7 of explant culture of bovine articular cartilage tissue. $+p<0.05$ using one-way 
ANOVA with Tukey's test. DMMB: dimethylmethylene blue; HAC: human articular cell; sGAG: sulfated glycosaminoglycan.

(a)
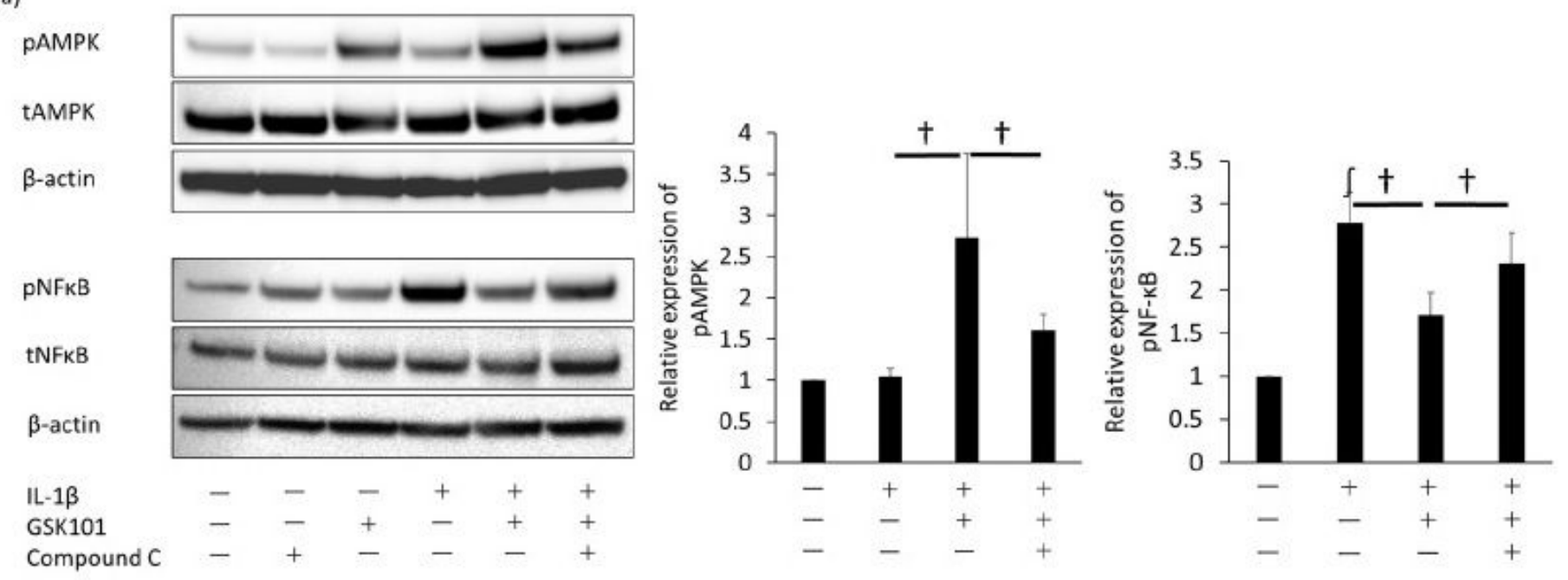

(b)
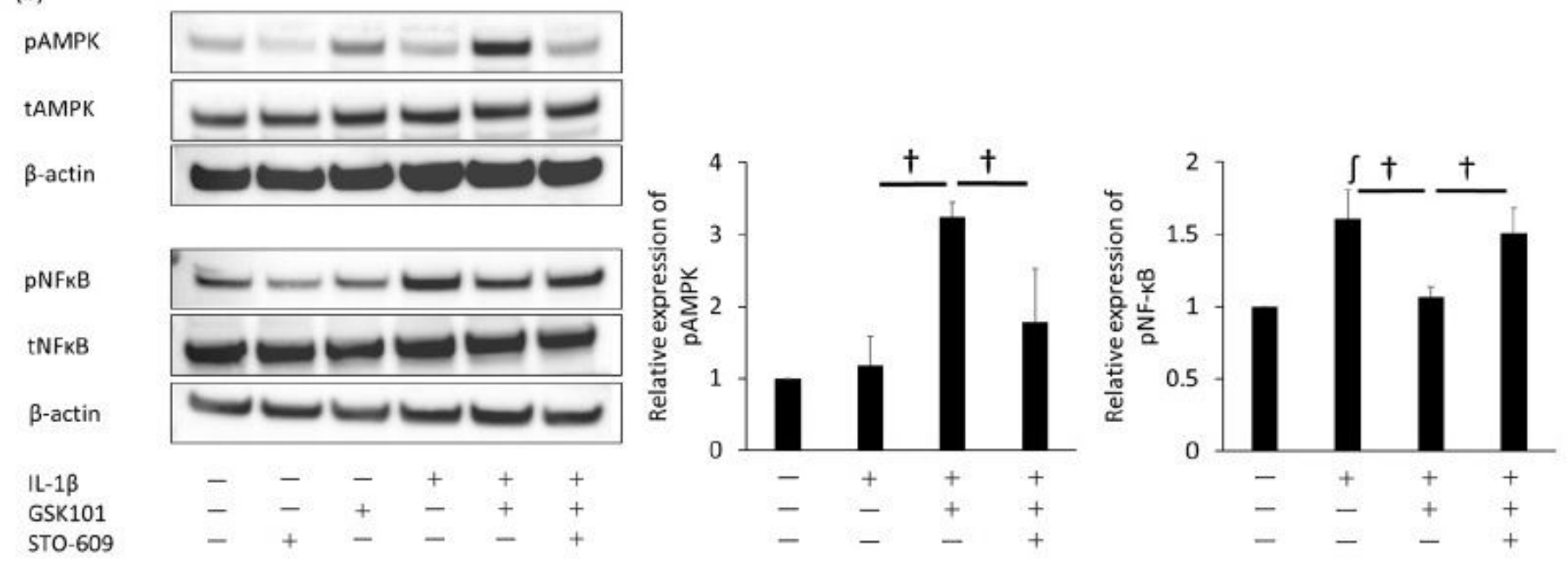

Figure 3

Inhibition of NF-KB phosphorylation by activation of the CaMKK/AMPK pathway Levels of phosphorylated AMPK and NF-KB in BACs treated with (a) IL-1 $\beta$, GSK101, and Compound C (25 $\mu M)$, and (b) IL-1 $\beta$, GSK101, and ST0-609 (5 $\mu \mathrm{M})$ for 30 minutes, as assessed by Western blot. Experiments were repeated three times $(n=3)$. Each group of $p N F \kappa B$, tNFKB and $\beta$-actin was derived from the same membrane. Each group of pAMPK, tAMPK and $\beta$-actin was derived from the same membrane. The membrane associated with NFKB and the membrane associated with AMPK were different while their protein samples were the same. $\int p<0.05$ compared to untreated control; $\uparrow p<0.05$ using one-way ANOVA with Tukey's test. BAC: bovine articular cell. 

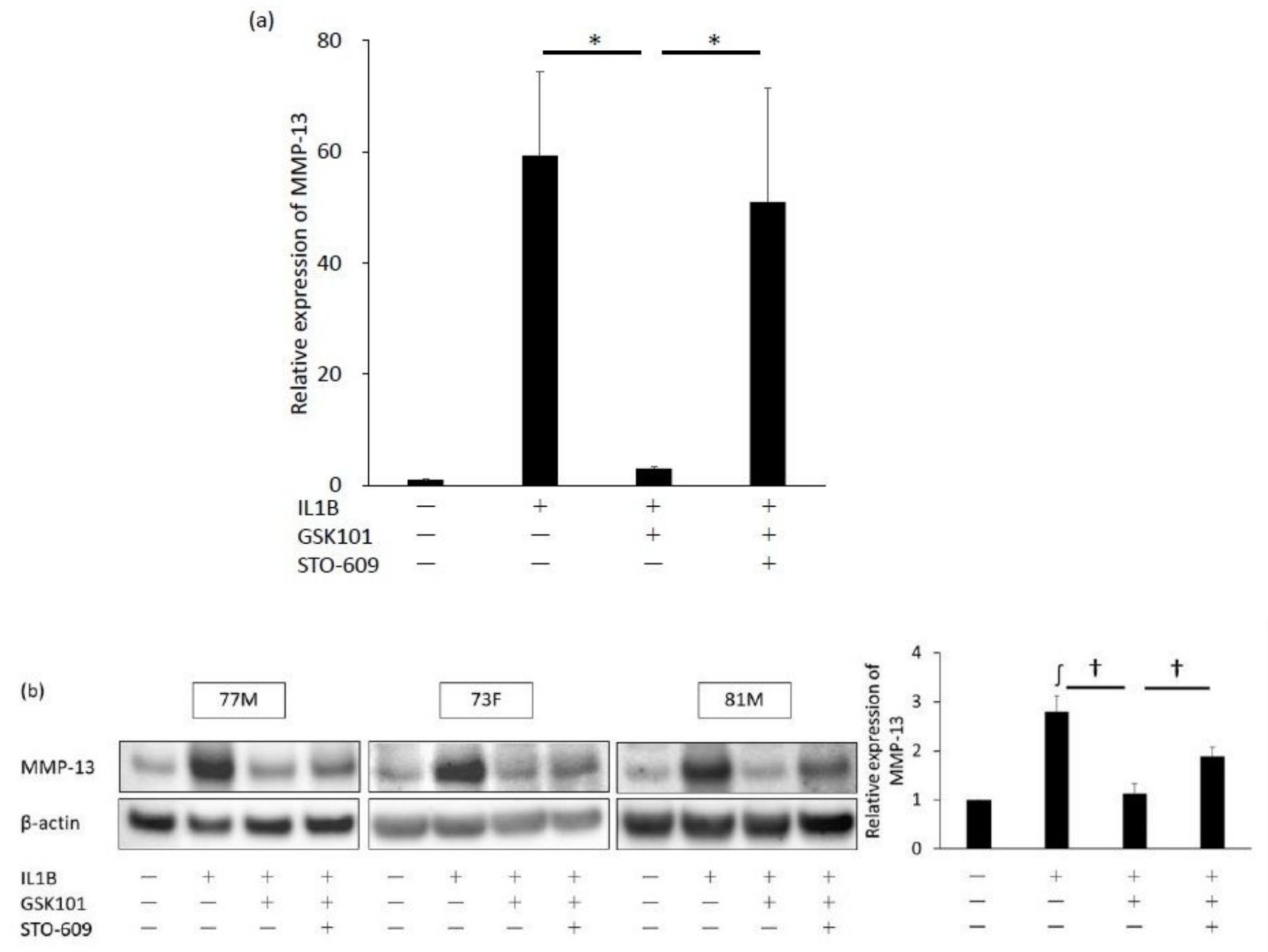

\section{Figure 4}

Inhibition of IL-1 $\beta$-induced cartilage damage via the TRPV4/CaMKK pathway (a) Real-time PCR analysis of relative expression of MMP-13 in BACs treated with IL-1 $\beta$, GSK101, and STO-609 for 12 hours. (b) Western blot analysis of relative expression of MMP-13 in HACs treated with IL-1 $\beta$, GSK101, and STO-609 for 48 hours. Experiments were repeated three times $(n=3)$. Each group of MMP-13 and $\beta$-actin was derived from the same membrane. $\int p<0.05$ compared to untreated control; $\uparrow p<0.05$ using one-way ANOVA with Tukey's test. BAC: bovine articular cell; HAC: human articular cell.

\section{Supplementary Files}

This is a list of supplementary files associated with this preprint. Click to download.

- Supplementarydata1.Allmembraneoffigure2..pdf 
- Supplementarydata2.Allmembraneoffigure3..pdf

- Supplementarydata3.Allmembraneoffigure4..pdf 